\title{
Ingénierie tissulaire osseuse en chirurgie buccale et maxillo-faciale : applications cliniques
}

\author{
Sylvain Catros ${ }^{1,2,3 \star}$, Fabien Guillemot ${ }^{1}$, Joëlle Amédée ${ }^{1}$, Jean-Christophe Fricainn ${ }^{1,2,3}$ \\ 1 Inserm U577 Biomatériaux et Réparation tissulaire, 16 rue Léo Saignat, Case 45, 33076 Bordeaux Cedex, France \\ 2 Faculté de Chirurgie dentaire, 16-20 cours de la Marne, 33076 Bordeaux, France \\ 3 Pôle d'Odontologie et de Santé buccale, Hôpital Pellegrin, 33000 Bordeaux, France
}

(Reçu le 22 août 2010, accepté le 7 septembre 2010)

Mots clés :

ingénierie tissulaire osseuse / scaffold / BMP / cellules souches mésenchymateuses / reconstruction osseuse
Key words:

bone tissue engineering / scaffold / BMP / mesenchymal stem cells / bone reconstruction
Résumé - L'ingénierie tissulaire osseuse a pour objectif général de repousser les limites des méthodes conventionnelles de régénération osseuse en utilisant les progrès des connaissances dans le domaine de la biologie, de l'ingénierie, de la physique ou encore de la robotique. Quatre éléments sont associés dans cette démarche d'ingénierie : un matériau support pour la croissance tissulaire (scaffold), des cellules autologues, des facteurs de croissance et une période de maturation in vitro ou in vivo. Dans la sphère buccale et maxillo-faciale, il s'agit de répondre au problème de la vascularisation des greffons de grande taille sur un terrain fragilisé et de remplacer les autogreffes osseuses qui peuvent provoquer une morbidité importante du site donneur.

L'objectif de cet article est une mise au point sur les applications cliniques humaines de l'ingénierie tissulaire osseuse pour la cavité buccale et la face. La méthode utilisée comporte une recherche bibliographique sur Pubmed et Medline avec des mots clés spécifiques. De plus, des critères d'inclusion et d'exclusion des articles recueillis ont été définis. Quarante-huit publications internationales ont été retenues et classées en fonction de leur domaine d'application. Il s'agissait de reconstruction mandibulaire (19 articles), de reconstruction osseuse dans le traitement des fentes faciales ( 6 articles) ou de régénération osseuse préimplantaire (23 articles). L'analyse de ces publications a montré qu'il s'agissait essentiellement d'études de faible puissance, à l'exception de 6 études prospectives randomisées dans le domaine de la régénération osseuse pré-implantaire. L'association d'un scaffold à des facteurs de croissance (BMPs) semble constituer la combinaison qui donne les meilleurs résultats. Pour la reconstruction mandibulaire, la méthode de prévascularisation in vivo des produits d'ingénierie tissulaire est une technique complexe mais prometteuse pour les patients en échec thérapeutique. L'ingénierie tissulaire osseuse a montré son efficacité dans la chirurgie pré-implantaire. Elle permettra peut-être bientôt d'envisager des techniques moins invasives.

\begin{abstract}
Bone tissue engineering in oral and maxillofacial surgery: clinical applications. Bone tissue engineering was developed to solve current limitations of conventional bone regeneration through an adequate combination of biology, engineer science, physics or robotics. There are 4 individual components necessary to define tissue engineering: the scaffold, the autologous cells, the growth factors and the maturation step (in vitro or in vivo). The objectives of oral and maxillofacial applications of bone tissue engineering are to improve vascularization of large osseous grafts and to avoid the morbidity of autografts harvest site. The aim of this article is to bring an update on human clinical applications of oral and maxillofacial bone tissue engineering. We did a bibliographic research in Pubmed and Medline databases with specific keywords. Inclusion and exclusion criteria were also defined. We finally retained 48 international articles, which covered 3 different fields. The subjects were mandibular reconstruction (19 articles), facial cleft treatment ( 6 articles) and bone regeneration related to dental implants ( 23 articles). Most of these articles were case reports and only 6 studies were prospective studies in the field of bone regeneration related to dental implants. Today, the best clinical results are obtained using a scaffold loaded with growth factors (BMPs). For mandibular reconstruction, in vivo maturation of tissue engineering products is a promising technique in selected patients. Bone tissue engineering is already an efficient technique in pre-implant bone surgery and could led to a lower morbidity of reconstructive procedures.
\end{abstract}

\footnotetext{
*Correspondance : sylvaincatros@hotmail.com
} 


$\begin{array}{ll}\text { Abréviations } & \\ \text { BMP } & \text { : bone morphogenetic protein } \\ \text { GDF-5 } & : \text { growth and differenciation factor -5 } \\ \text { HA } & : \text { hydroxyapatite } \\ \text { HA-TCP } & : \text { hydroxyapatite-phosphate tricalcique } \\ \text { IT } & : \text { ingénierie tissulaire } \\ \text { PIT } & \text { : produit d'ingénierie tissulaire } \\ \text { PLGA } & : \text { platelet rich fibrin } \\ \text { PRF } & : \text { platelet rich plasma } \\ \text { PRP } & : \text { human recombinant } \\ \text { rhBMP-2 } & \text { bone morphogenetic protein }-2 \\ \text { rhBMP-7 } & \text { : human recombinant } \\ \text { rhGDF-5 } & \text { bone morphogenetic protein }-7 \\ \text { TCP } & \text { recombinant growh } \\ \text { TGF } & \text { and differenciation factor }-5 \\ & : \text { transforming growth factor }\end{array}$

L'ingénierie tissulaire (IT) a été définie comme «l'ensemble des techniques et des méthodes s'inspirant des principes de l'ingénierie et des sciences de la vie, utilisées pour développer des substituts biologiques pouvant restaurer, maintenir ou améliorer les fonctions des tissus »[1]. Le principe général est d'associer une matrice tridimensionnelle (scaffold), avec des cellules autologues et des facteurs de croissance pour reconstruire un nouveau tissu hybride (Fig. 1).

Les applications cliniques chez l'homme existent déjà pour la peau depuis les années 1990 [2]. Plus récemment, des tissus produits par IT ont été développés pour les muscles squelettiques, le cœur, les gros vaisseaux ou le tissu nerveux [3]. Un des objectifs de la médecine régénératrice est de développer des substituts d'organes entiers (vessie, rein, pancréas) pour faire face aux demandes croissantes de greffons.

L'IT osseuse a été développée pour repousser les limites des méthodes conventionnelles de traitement des pertes de substance osseuses, en particulier la morbidité induite par les autogreffes et l'absence d'intégration des greffes de grande taille.

L'objectif de cette mise au point est de rechercher quelles sont les applications cliniques humaines actuelles et quel est le bénéfice de l'ingénierie tissulaire osseuse dans la sphère buccale et maxillo-faciale.

\section{Matériel et méthodes}

\section{Méthode de recherche}

Les bases de données Medline et Pubmed ont été consultées sur la période 1999-2010 pour les articles en anglais ou en français, avec les mots clés suivants :

- mots clés MESH (Medical Subject Headings) : «tissue engineering/methods » or «tissue engineering/utilization » or " tissue engineering/veterinary » and " facial bones/surgery » or « facial bones/transplantation » ;

- autres mots clés utilisés : « mandible reconstruction human » / « mandible reconstruction growth factor human »/

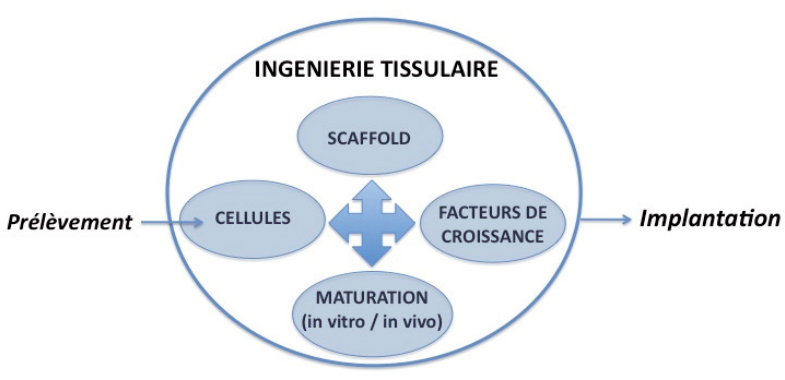

Fig. 1. Les étapes nécessaires à la construction d'un tissu par ingénierie tissulaire.

Fig. 1. Tissue engineering steps for tissue repair.

« mandible reconstruction cells human » / « sinus lift tissue engineering » / « sinus lift growth factor »/ BMP / « maxillary bone tissue engineering 》 / " sinus lift cells».

Enfin une recherche manuelle sur les références des articles sélectionnés a été réalisée pour croiser les informations obtenues. Les articles sélectionnés ont ensuite été regroupés en fonction du domaine clinique d'application.

\section{Critères d'inclusion}

Les études cliniques originales ou les cas rapportés utilisant un produit d'ingénierie tissulaire (PIT) osseuse avec au moins deux des quatre critères suivants :

- matrice organique ou inorganique;

- cellules autologues prélevées, amplifiées et induites;

- facteurs de croissance;

- maturation des PIT osseuse (in vivo ou in vitro).

\section{Critères d'exclusion}

- Revues de littérature.

- Études n'utilisant pas au moins deux des critères décrits précédemment.

- Études utilisant le PRP (Platelet Rich Plasma) ou le PRF (Platelet Rich Fibrin) comme facteur de croissance.

- Études utilisant les autogreffes ou les lambeaux seuls.

\section{Résultats}

Quarante-huit articles concernant la reconstruction osseuse dans la sphère maxillo-faciale ont été retenus et analysés en fonction des critères définis. Trente-cinq études correspondent à des rapports de cas, 7 articles à des études prospectives non contrôlées et 6 articles à des études prospectives randomisées de faible puissance.

Seule l'étude de cas de Mesimäki et al. [4] associait les quatre critères d'inclusion. Dans 16 articles, trois des critères d'inclusion étaient présents (scaffold + cellules + maturation 
ou scaffold + BMP + maturation). Dans 31 articles, deux critères d'inclusion étaient présents (scaffold et cellules autologues différenciées ou scaffold et facteurs de croissance) (Tabs. I, II et III).

Le scaffold était utilisé dans tous les cas. Les matériaux étaient des phosphates de calcium synthétiques (12 cas), des polymères tressés (9 cas), des allogreffes ( 5 cas), des xénogreffes d'origine bovine ( 5 cas), des autogreffes (2 cas), du collagène d'origine bovine (13 cas) ou un gel de fibrine autologue ou non (12 cas). Dans certains cas, plusieurs scaffolds étaient utilisés. Les cellules utilisées étaient le plus souvent issues de moelle osseuse iliaque (15 cas). Elles pouvaient aussi provenir du périoste ( 7 cas), du tissu adipeux (2 cas) ou bien de la pulpe dentaire (1 cas). Les facteurs de croissance utilisés étaient des BMPs d'origine humaine ou bovine dans les premières études ( 3 cas). Puis, des formes recombinantes ont été utilisées (rhBMP-2 : 10 cas; rhBMP-7 : 5 cas, rhGDF : 1 cas).

Dix-neuf articles décrivaient la reconstruction de grands défauts osseux maxillaires ou mandibulaires par ingénierie tissulaire (Tab. I). Il s'agissait toujours de cas complexes, chez des patients en échec thérapeutique avec les méthodes conventionnelles de greffes osseuses ou de lambeaux autologues. Le site receveur était souvent constitué d'un os hypovascularisé et hypocellularisé par la radiothérapie, ou infecté (ostéomyélites) (Tab. I) [4-21].

Les scaffolds utilisés étaient des phosphates de calcium, des cages en titane, des éponges de collagène, de la fibrine ou des polymères (dacron, polycaprolactone). Dans 5 cas, des cellules mésenchymateuses indifférenciées étaient induites vers un phénotype ostéoblastique in vitro avant implantation et, dans 5 cas, des greffes osseuses ou de la moelle osseuse autologue ont été utilisées. Les BMPs ont été utilisées dans 12 cas sur 18.

La maturation du PIT a été réalisée in vivo dans 7 cas chez l'homme depuis le premier cas publié en 1999 [5]. Quatre cas ont été réalisés à la mandibule [5-8] et un cas au maxillaire [4]. Le principe était de placer l'assemblage construit par IT à proximité d'un muscle, qui est un site favorable pour le développement de la vascularisation (muscle grand dorsal $[5,6,8,13]$, grand pectoral [7] ou grand droit de l'abdomen [4]). Cette étape a permis le dépôt de tissu osseux et la formation de néo-vaisseaux dans les matériaux implantés. Après plusieurs mois de maturation, le greffon était déplacé vers le site receveur grâce à un lambeau pédiculé (1 cas) ou libre (4 cas). Dans un cas, le greffon a subi une maturation in vitro avant son implantation (après ensemencement des cellules sur le scaffold) [14].

Deux articles $[9,22]$ ont présenté des cas de reconstruction mandibulaire par la technique des membranes induites, initialement décrite pour la reconstruction des os longs $[23,24]$. Cette méthode a été utilisée à la mandibule pour traiter des cas d'ostéomyélites et d'ostéoradionécroses $[9,19]$. Le principe était d'induire dans un premier temps chirurgical la formation d'une membrane synoviale autour du défaut osseux grâce à l'interposition d'une entretoise de ciment polyméthylmétacrylate, puis de combler la perte de substance par une autogreffe ou un biomatériau dans un second temps.

Six rapports de cas (Tab. II) [25-30] présentaient la reconstruction de fentes congénitales palatines ou faciales par ingénierie tissulaire. Les auteurs ont souvent utilisé l'IT en complément de distractions osseuses [25, 26, 30]. Les scaffolds utilisés dans les fentes étaient soit des éponges de collagène, soit de la fibrine (préparée avec du PRP), soit un phosphate de calcium. Des ostéoblastes issus de la moelle osseuse ont été ajoutés aux scaffolds dans 3 cas. Les BMPs ont été utilisées dans un seul cas. La maturation du PIT n'a jamais été utilisée. La reconstruction osseuse obtenue après traitement était quasi complète ou complète, ce qui a permis l'éruption normale des dents retenues dans plusieurs cas.

Dans 23 articles, l'ingénierie tissulaire osseuse a été utilisée pour la régénération osseuse pré-implantaire et parodontale (Tab. III) [31-53]. Il s'agissait de l'élévation du plancher des sinus maxillaires (15 articles), de la régénération de défauts péri-implantaires ( 3 articles), de l'augmentation osseuse alvéolaire verticale ou transversale ( 4 articles) ou du traitement de défauts osseux angulaires parodontaux (1 article). Dans ces indications, les scaffolds utilisés étaient soit des matériaux non déformables (phosphates de calcium, xénogreffes : 9 cas), soit des hydrogels (fibrine : 6 cas), soit des éponges (collagène, PLGA ou copolymère acide polyglycolique + acide polylactique : 11 cas). Dans 17 cas sur 23, des cellules mésenchymateuses induites vers un phénotype ostéoblastique (moelle osseuse, tissu adipeux, pulpe dentaire) ont été ensemencées dans les scaffolds. Les BMPs (rhBMP2) ont été utilisées dans 5 cas pour imprégner des scaffolds et dans un cas, le GDF-5 a été greffé de façon covalente sur un phosphate de calcium. La maturation in vitro a été utilisée dans 10 cas. Dans 3 articles [31-53], un protocole commercial d'ingénierie tissulaire osseuse a été mis en œuvre (protocole Bioseed ${ }^{\circledR}$ et protocole Biotissue ${ }^{\circledR}$ ). Il s'agissait d'un scaffold de fibres de PLGA ensemencé avec des ostéoblastes issus du périoste, et mis en maturation in vitro pendant 7 jours avant implantation. Les résultats étaient inconstants mais globalement positifs avec cette méthode.

\section{Discussion}

La méthode de recherche bibliographique a suivi les recommandations du Guide d'analyse de la littérature et gradation des recommandations de l'HAS [54]. Un grand nombre d'articles avec un faible niveau de preuve a été retenu car il existait peu d'études humaines. En effet, de nombreuses études existaient sur des modèles animaux mais peu d'études ont été publiées chez l'homme. Cela peut s'expliquer par la fiabilité importante des méthodes conventionnelles d'autogreffes dans la prise en charge de la majorité des pertes osseuses maxillo-faciales et alvéolaires et leur morbidité modérée $[55,56]$. La relative nouveauté, la complexité et le coût de certaines méthodes d'IT peuvent expliquer le peu de cas publiés. De plus, 8 équipes ont publié environ la moitié des articles retenus, ce qui montre qu'il existe relativement peu 
Tableau I. Chirurgie osseuse reconstructrice maxillaire ou mandibulaire. [ADSC-OB : cellules mésenchymateuses issues de tissu adipeux, induites vers un phénotype ostéoblastique ; HA-TCP : hydroxyapatite - phosphate tricalcique; PRP : plasma riche en plaquettes ; HBMSC-OB : cellules mésenchymateuses issues de moelle osseuse, induites vers un phénotype ostéoblastique; rhBMP-2 : bone morphogenetic protein -2 (recombinante); rhBMP-7 : bone morphogenetic protein -7 (recombinante)].

Table I. Maxilla and mandible bone reconstruction. [ADSC-OB: adipose derived stromal cells, induced to osteoblastic phenotype; HA-TCP: hydroxyapatite - tricalcium phosphate; HBMSC-OB: human bone marrow stromal cells, induced to osteoblastic phenotype; PRP: platelet rich plasma; rhBMP-2: bone morphogenetic protein -2 (recombinant); rhBMP-7: bone morphogenetic protein -7 (recombinant)].

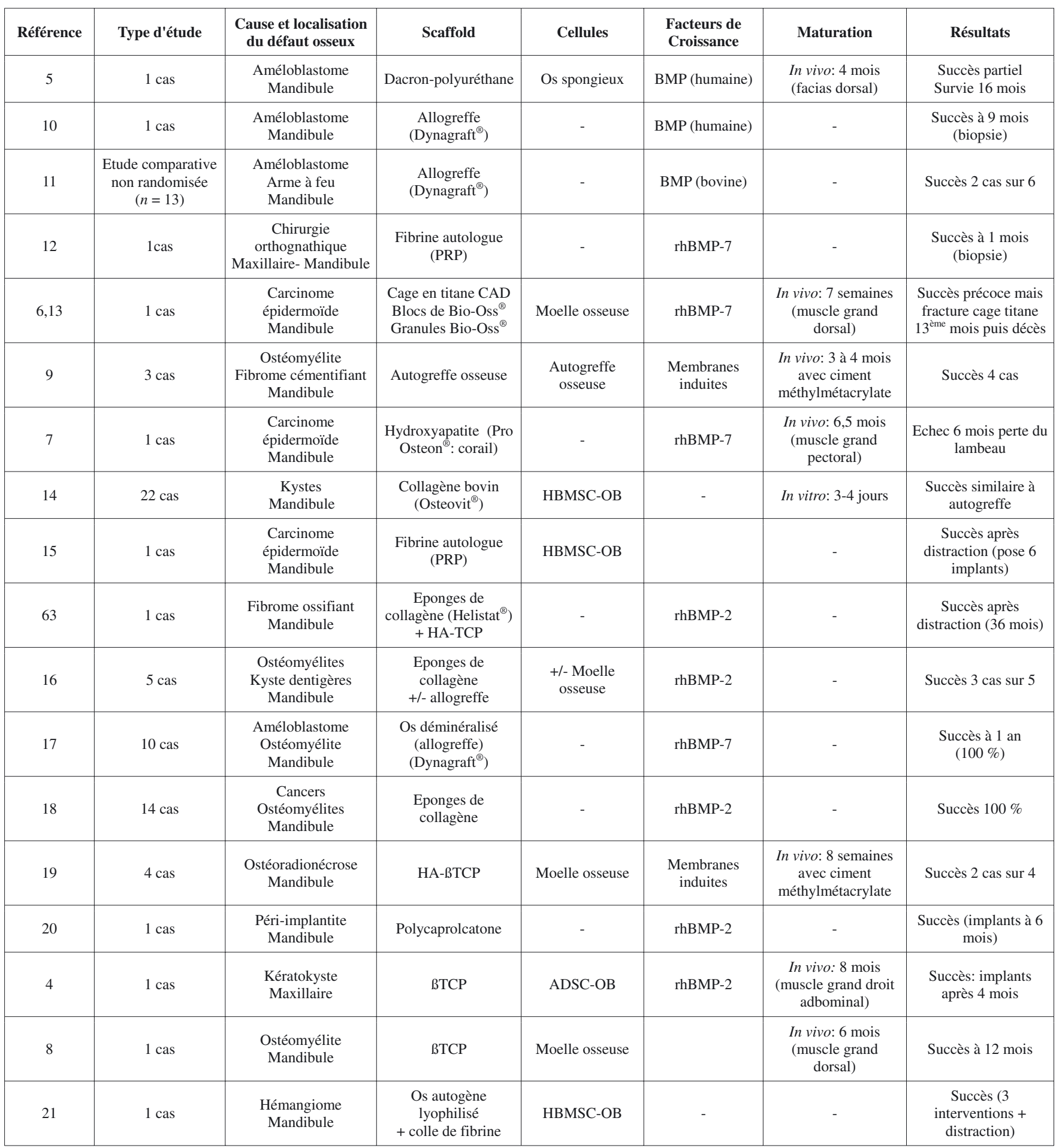


Tableau II. Chirurgie osseuse reconstructrice pour les fentes faciales. [HBMSC : cellules mésenchymateuses issues de moelle osseuse ; HBMSCOB : cellules mésenchymateuses issues de moelle osseuse, induites vers un phénotype ostéoblastique; PRP : plasma riche en plaquettes; rhBMP-2 : bone morphogenetic protein -2 (recombinante)].

Table II. Facial clefts bone reconstruction. [HBMSC: human bone marrow stromal cells; HBMSC-OB: human bone marrow stromal cells, induced to osteoblastic phenotype; PRP: platelet rich plasma; rhBMP-2: bone morphogenetic protein -2 (recombinant)].

\begin{tabular}{|c|c|c|c|c|c|c|c|}
\hline Référence & \begin{tabular}{|c|} 
Type \\
d'étude
\end{tabular} & Objectif & Scaffold & Cellules & $\begin{array}{l}\text { Facteurs de } \\
\text { croissance }\end{array}$ & Maturation & Résultats \\
\hline 25,26 & 1 cas & $\begin{array}{c}\text { Reconstruction fente faciale } \\
\text { complexe + hypoplasie } \\
\text { mandibulaire }\end{array}$ & $\begin{array}{c}\text { Eponge de collagène } \\
\left(\text { Helistat }^{\circledR}\right)\end{array}$ & - & rhBMP-2 & - & $\begin{array}{l}\text { Suivi } 4,5 \text { ans } \\
\text { Reconstruction } \\
\text { partielle }\end{array}$ \\
\hline 30 & 50 cas & $\begin{array}{l}\text { Réparation fentes par rhBMP-2, } \\
\text { sans autogreffe }\end{array}$ & Eponge de collagène & - & rhBMP-2 & - & $\begin{array}{l}\text { Succès } 49 / 50 \\
\text { +/- distraction }\end{array}$ \\
\hline 27 & 1 cas & $\begin{array}{l}\text { Reconstruction fente alvéolo- } \\
\text { palatine }\end{array}$ & $\begin{array}{l}\text { Fibrine autologue } \\
\text { (PRP) }\end{array}$ & HBMSC-OB & & - & $\begin{array}{l}\text { Régénération } \\
79 \% \text { du défaut }\end{array}$ \\
\hline 28 & 1 cas & $\begin{array}{l}\text { Reconstruction fente alvéolo- } \\
\text { palatine }\end{array}$ & $\begin{array}{l}\text { Eponge de collagène } \\
\left(\text { Ostéovit }^{\circledR}\right)\end{array}$ & HBMSC & - & 3 à 4 jours & $\begin{array}{l}\text { Fermeture } \\
\text { complète fente } \\
\text { (Rx } 18 \text { mois) }\end{array}$ \\
\hline 29 & 2 cas & $\begin{array}{l}\text { Reconstruction fente alvéolo- } \\
\text { palatine }\end{array}$ & $\begin{array}{l}\text { Os deminéralisé + } \\
\text { sulfate de calcium } \\
\quad\left(\text { Osteoset }{ }^{\circledR}\right)\end{array}$ & HBMSC-OB & - & - & $\begin{array}{l}\text { Régénération } \\
66 \% \text { et } 75 \% \text { du } \\
\text { défaut }\end{array}$ \\
\hline
\end{tabular}

de groupes utilisant l'IT en reconstruction osseuse maxillofaciale.

Deux paramètres sur quatre définissant l'IT étaient nécessaires pour retenir un article (scaffold/cellules/facteurs de croissance/maturation in vivo ou in vitro). La notion de manipulation est indispensable car elle sous-entend une organisation des rapports entre les différents composants, afin de contrôler la maturation et la régénération tissulaire [1]. Les études utilisant le PRP ou le PRF comme facteurs de croissance n'ont pas été retenues car des controverses persistent sur l'effet ostéo-inducteur réel de ces produits [57]. Les méthodes de préparation varient et les résultats sont parfois très différents en fonction du protocole utilisé [58]. Cependant, les articles utilisant le PRP comme scaffold ont été inclus.

L'intérêt de l'IT en chirurgie buccale et maxillo-faciale est fonction de l'indication.

Dans la reconstruction mandibulaire, la principale limite des méthodes conventionnelles de reconstruction (lambeaux autologues libres ou pédiculés) est la mauvaise vascularisation locale des greffons. Elle résulte des caractéristiques anatomiques locales (vascularisation terminale) et de la radiothérapie qui altère de façon durable la vascularisation locale et la composante cellulaire du tissu osseux. L'objectif principal de l'IT dans la reconstruction mandibulaire est la prévascularisation et la précellularisation des greffons. La méthode de maturation in vivo des PIT a permis dans plusieurs cas de rétablir une néovascularisation qui a persisté après implantation (Tab. I). Dans le traitement des fentes faciales et dans la régénération osseuse pré-implantaire et parodontale, c'est essentiellement la morbidité liée au site donneur d'os autogène qui a motivé le recours à l'IT (Tab. II et III) [59]. Il faut remarquer que 51 des 55 cas de fentes faciales traités par ingénierie tissulaire provenaient de la même équipe $[25,26,30]$, ce qui montre que l'IT reste marginale dans cette indication.
Les scaffolds utilisés dans les cas de reconstruction mandibulaire étaient des phosphates de calcium (os bovin déprotéiné, HA, HA-TCP, ßTCP) stabilisés par des vis d'ostéosynthèse [7] ou par des cages en titane préformées $[4,6,8]$. Dans le cas publié par Orringer et al. [5], l'absence de propriétés mécaniques du scaffold (membranes de dacron imprégnées de BMP) n'a pas permis de contrôler la morphologie de la reconstruction, ce qui a compliqué la réhabilitation prothétique de la patiente. Les complications observées par Warnke et al. [6] et de Heliotis et al. [7] sont également directement en rapport avec une défaillance du scaffold (fracture de la cage en titane ou difficultés de fixation du matériau). La résistance mécanique et la stabilité immédiate des reconstructions sont donc un point crucial pour le succès de l'IT en chirurgie maxillo-faciale.

Dans les fentes faciales, des matériaux injectables ou très malléables ont été choisis (fibrine, collagène), afin de faciliter leur insertion.

Dans les régénérations osseuses pré-implantaires, les scaffolds étaient très variés (fibrine, collagène, PLGA, phosphates de calcium). Ils avaient globalement des propriétés mécaniques modérées.

Des ostéoblastes dérivés de moelle osseuse (3 cas) ou de tissu adipeux (1 cas), ensemencés dans des scaffolds, ont été utilisés dans les reconstructions mandibulaires. Ces cas ne confirment pas réellement l'intérêt de l'association cellulesscaffold dans cette indication. Le recours aux cellules complique la procédure (prélèvement, culture cellulaire et ensemencement) et allonge la durée globale de la reconstruction. L'utilisation des cellules semble plus pertinente en présence de facteurs de croissance.

Dans la reconstruction des fentes faciales, les cellules mésenchymateuses issues de moelle osseuse induites ou non vers un phénotype ostéoblastique ont été utilisées dans quatre cas 
Tableau III. Chirurgie osseuse reconstructrice pré-implantaire. [ADSC-OB : cellules mésenchymateuses issues de tissu adipeux, induites vers un phénotype ostéoblastique; $\mathrm{DPC}-\mathrm{OB}$ : cellules de la pulpe dentaire induites vers un phénotype ostéoblastique; GDF-5: growth and differenciation factor -5 ; HA : hydroxyapatite; HA-TCP : hydroxyapatite - phosphate tricalcique ; HBMSC : cellules mésenchymateuses issues de moelle osseuse; HBMSC-OB : cellules mésenchymateuses issues de moelle osseuse, induites vers un phénotype ostéoblastique ; PDSC-0B : cellules mésenchymateuses issues du périoste, induites vers un phénotype ostéoblastique; PLGA : copolymère acide polyglycolique + acide polylactique; PRP : plasma riche en plaquettes; rhBMP-2 : bone morphogenetic protein -2 (recombinante); ROG : régénération osseuse guidée].

Table III. Bone reconstruction for dental implant surgery. [ADSC-OB: adipose derived stromal cells, induced to osteoblastic phenotype; DPC-OB: dental pulp cells, induced to osteoblastic phenotype; GDF-5: growth and differenciation factor 5; HA: hydroxyapatite; HA-TCP: hydroxyapatite tricalcium phosphate; HBMSC-OB: human bone marrow stromal cells, induced to osteoblastic phenotype; HBMSC-OB: human bone marrow stromal cells; PDSC-OB: periosteal derived stromal cells, induced to osteoblastic phenotype; PLGA: poly (lactic-co-glycolic acid); PRP: platelet rich plasma; rhBMP-2: bone morphogenetic protein -2 (recombinant); ROG: guided bone regeneration].

\begin{tabular}{|c|c|c|c|c|c|c|c|}
\hline Référence & Type d'étude & Objectif & Scaffold & Cellules & $\begin{array}{l}\text { Facteurs de } \\
\text { Croissance }\end{array}$ & Maturation & Résultats \\
\hline 49 & \begin{tabular}{|c|} 
Etude \\
prospective non \\
contrôlée \\
$(n=12)$
\end{tabular} & $\begin{array}{l}\text { Sécurité et efficacité } \\
\text { des BMPs pour ROG } \\
\text { pré-implantaire }\end{array}$ & Éponge de collagène & - & rhBMP-2 & - & $\begin{array}{c}\text { Pas d'effet néfaste local } \\
\text { ou général des BMPs } \\
\text { Succès implantaire à } 3 \\
\text { ans }\end{array}$ \\
\hline 50 & $\begin{array}{c}\text { Etude contrôlée } \\
\text { randomisée } \\
(n=11,34 \\
\text { implants })\end{array}$ & $\begin{array}{l}\text { Intérêt de l'ajout de } \\
\text { BMP pour ROG } \\
\text { péri-implantaire }\end{array}$ & $\mathrm{BioOss}^{\circledR}$ & - & rhBMP-2 & - & $\begin{array}{c}\text { Augmentation volume os } \\
\text { néoformé et diminution } \\
\text { durée cicatrisation avec } \\
\text { rhBMP-2 }\end{array}$ \\
\hline 35 & 2 cas & Sinus lift par IT & $\begin{array}{l}\text { Membrane Ethisorb }{ }^{\circledast} \\
+ \text { fibrine }\left(\text { Tissucoll }^{\circledR} \text { ) }\right.\end{array}$ & PDSC-OB & - & $\begin{array}{l}\text { In vitro: } \\
1 \text { semaine }\end{array}$ & $\begin{array}{c}\text { Succès }(\mathrm{Rx}+\text { biopsie }) \\
\text { Insertion des implants à } 4 \\
\text { mois }\end{array}$ \\
\hline 36 & \begin{tabular}{|c|} 
Etude \\
prospective non \\
contrôlée \\
$(n=27)$
\end{tabular} & Sinus lift par IT & Membrane Ethisorb $^{\circledR}$ & PDSC-OB & - & $\begin{array}{l}\text { In vitro: } \\
1 \text { semaine }\end{array}$ & $\begin{array}{l}\text { Succès } 18 \text { cas } \\
\text { Echec } 9 \text { cas }\end{array}$ \\
\hline 52 & 3 cas & $\begin{array}{c}\text { ROG péri- } \\
\text { implantaire par } \\
\text { substitut IT injectable }\end{array}$ & $\begin{array}{l}\text { Fibrine autologue } \\
\text { (PRP) }\end{array}$ & HBMSC-OB & & - & $\begin{array}{c}\text { Succès sur } 3 \text { cas (10 } \\
\text { implants) }\end{array}$ \\
\hline 51 & $\begin{array}{l}\text { Etude contrôlée } \\
\text { randomisée } \\
(n=48)\end{array}$ & $\begin{array}{l}\text { Détermination de la } \\
\text { concentration } \\
\text { nécessaire de } \\
\text { rhBMP2 pour sinus } \\
\text { lift }\end{array}$ & $\begin{array}{c}\text { Éponge de collagène } \\
\left(\text { Helistat }^{\circledR}\right)\end{array}$ & - & rhBMP-2 & - & $\begin{array}{l}\text { BMP-2: } 80 \% \text { succès } \\
\text { Concentration BMP-2 } \\
\text { idéale }=1,5 \mathrm{mg} / \mathrm{ml}\end{array}$ \\
\hline 37 & 1 cas & $\begin{array}{c}\text { Augmentation } \\
\text { osseuse transversale } \\
\text { mandibule }\end{array}$ & $\begin{array}{c}\text { PLGA (Vicryl); } \\
\text { Protocole Bioseed }^{\circledast}\end{array}$ & PDSC-OB & - & $\begin{array}{l}\text { In vitro: } \\
5 \text { semaines } \\
\text { puis greffe }\end{array}$ & $\begin{array}{c}\text { Succès: pose } 2 \text { implants à } \\
6 \text { mois }\end{array}$ \\
\hline 38 & \begin{tabular}{|c|} 
Etude \\
comparative \\
non randomisée \\
$(n=13)$
\end{tabular} & Sinus lift & $\begin{array}{c}\text { Xenogreffe } \\
\left(\text { BioOss }^{\circledR}\right) \\
\text { Eponge de collagène } \\
\left(\text { Lyostypt }^{\circledR}\right)\end{array}$ & $\begin{array}{l}\text { HBMSC-OB } \\
\text { PDSC-OB }\end{array}$ & - & $\begin{array}{l}\text { In vitro: } \\
7 \text { à } 40 \text { jours }\end{array}$ & $\begin{array}{l}\text { IT supérieure à } \\
\text { autogreffe }\end{array}$ \\
\hline 45 & \begin{tabular}{|c|} 
Etude \\
comparative \\
non randomisée \\
$(n=20)$
\end{tabular} & $\begin{array}{l}\text { Comparaison } \\
\text { résorption os } \\
\text { autogène vs os IT } \\
\text { dans sinus lift }\end{array}$ & $\begin{aligned} & \text { PLGA (Ethicon) } \\
+ & \text { fibrine }\left(\text { Tissucol }^{\circledR}\right)\end{aligned}$ & HBMSC-OB & - & $\begin{array}{l}\text { In vitro: } \\
\text { 6-9 jours }\end{array}$ & $\begin{array}{l}29 \% \text { résorption os } \\
\text { autogène } \\
90 \% \text { résorption os IT }\end{array}$ \\
\hline 43 & 6 cas & IT pour sinus lift & HA-TCP & HBMSC-OB & - & - & $\begin{array}{l}93 \% \text { succès implants } \\
\text { dans os IT }\end{array}$ \\
\hline 42 & 12 cas & IT pour sinus lift & $\begin{array}{l}\text { Fibrine autologue } \\
\text { (PRP) }\end{array}$ & HBMSC-OB & & - & $\begin{array}{c}\text { Gain } 8 \mathrm{~mm} \text { hauteur os et } \\
100 \% \text { succès implants } \\
(n=41) \text { à } 2-6 \text { ans }\end{array}$ \\
\hline 41 & $8 \mathrm{cas}$ & $\begin{array}{l}\text { Défauts osseux } \\
\text { alvéolaires } \\
\text { transversaux et } \\
\text { verticaux }\end{array}$ & $\begin{array}{l}\text { Phosphate de calcium } \\
\left.\text { (Biomatrix }{ }^{\circledR}\right)\end{array}$ & ADSC-OB & & $\begin{array}{l}\text { In vitro: } \\
7 \text { jours }\end{array}$ & $\begin{array}{l}\text { Succès } 8 \text { cas: pose des } \\
\text { implants à } 3 \text { mois }\end{array}$ \\
\hline
\end{tabular}


Tableau III. Suite.

Table III. Continued.

\begin{tabular}{|c|c|c|c|c|c|c|c|}
\hline Référence & Type d'étude & Objectif & Scaffold & Cellules & $\begin{array}{l}\text { Facteurs de } \\
\text { Croissance }\end{array}$ & Maturation & Résultats \\
\hline 53 & 6 cas & $\begin{array}{l}\text { ROG } \\
\text { péri-implantaire }\end{array}$ & HA $\left(\right.$ Proosteon $\left.^{\circledR}\right)$ & HBMSC-OB & - & $\begin{array}{l}\text { In vitro: } \\
7 \text { jours }\end{array}$ & $\begin{array}{c}1 \text { seul cas de néo- } \\
\text { formation osseuse par IT } \\
\text { sur } 6 \text { patients }\end{array}$ \\
\hline 39 & $\begin{array}{l}\text { Etude contrôlée } \\
\text { randomisée } \\
(n=30)\end{array}$ & $\begin{array}{l}\text { RTG défauts osseux } \\
\text { parodontaux }\end{array}$ & $\begin{array}{c}\text { HA } \\
+ \text { Fibrine }(\mathrm{PRP})\end{array}$ & PDSC-OB & & - & $\begin{array}{l}\text { Meilleurs résultats avec } \\
\text { IT (gain d'attache } \\
\text { clinique et comblement } \\
\text { défauts infra-osseux) }\end{array}$ \\
\hline 32 & 3 cas & Sinus lift & $\begin{array}{l}\text { Fibrine }\left(\text { Tissucoll }{ }^{\circledR} \text { ) }\right. \\
\text { PLGA (Ethicon) }\end{array}$ & PDSC-OB & - & $\begin{array}{l}\text { In vitro: } \\
21 \text { jours }\end{array}$ & $\begin{array}{l}100 \% \text { Succès } \\
\text { Os mature à } 6 \text { mois }\end{array}$ \\
\hline 44 & 14 cas & $\begin{array}{c}\text { Sinus lift } \\
\text { Greffe en onlay }\end{array}$ & $\begin{array}{l}\text { Fibrine autologue } \\
\text { (PRP) }\end{array}$ & HBMSC-OB & & - & $100 \%$ Succès \\
\hline 40 & $\begin{array}{l}\text { Etude contrôlée } \\
\text { randomisée } \\
(n=7)\end{array}$ & $\begin{array}{c}\text { Régénération alvéole } \\
(38-48)\end{array}$ & Eponges de collagène & $\begin{array}{l}\text { DPC-OB } \\
(18-28)\end{array}$ & - & - & $\begin{array}{l}\text { Régénération complète } \\
\text { des alvéoles avec IT: } \\
\text { résultat supérieur à celui } \\
\text { du contrôle }\end{array}$ \\
\hline 46 & $\begin{array}{l}\text { Etude } \\
\text { prospective non } \\
\text { contrôlée } \\
(n=22)\end{array}$ & Sinus lift & $\begin{array}{l}\text { Xénogreffe } \\
\left(\text { BioOss }^{\circledR}\right)\end{array}$ & HBMSC-OB & - & - & $\begin{array}{l}\text { Suffisant pour pose } \\
\text { d'implant, mais } \\
\text { résorption à } 6 \text { et } 12 \text { mois }\end{array}$ \\
\hline 33 & 5 cas & $\begin{array}{c}\text { Sinus lift } \\
\left(\text { comparaison }^{\circledR}\right. \\
\text { Biocoral }^{\circledR} \text { seul vs IT) }\end{array}$ & PLGA (Ethisorb ${ }^{\circledR}$ ) & HBMSC-OB & - & $\begin{array}{l}\text { In vitro: } \\
6 \text { à } 9 \text { jours }\end{array}$ & $\begin{array}{c}\text { Succès des } 2 \text { techniques } \\
\text { mais densité supérieure } \\
\text { avec HA }\end{array}$ \\
\hline 31 & $\begin{array}{l}\text { Etude } \\
\text { prospective } \\
\text { contrôlée } \\
\text { randomisée } \\
(n=160)\end{array}$ & $\begin{array}{c}\text { Sinus lift: (autogreffe } \\
\text { vs IT) }\end{array}$ & Eponge de collagène & - & rh-BMP-2 & - & $\begin{array}{c}\text { Reconstruction osseuse } \\
\text { équivalente avec les } 2 \\
\text { méthodes }\end{array}$ \\
\hline 34 & $\begin{array}{c}\text { Etude } \\
\text { prospective non } \\
\text { contrôlée } \\
(n=35)\end{array}$ & $\begin{array}{c}\text { Sinus lift: (autogreffe } \\
\text { vs IT) }\end{array}$ & PLGA (Ethicon ${ }^{\circledR}$ ) & PDSC-OB & - & 8 semaines & $\begin{array}{l}\text { Autogreffe }+ \text { efficace que } \\
\text { IT pour sinus lift }\end{array}$ \\
\hline 47 & $\begin{array}{l}\text { Etude contrôlée } \\
\text { randomisée } \\
(n=31)\end{array}$ & $\begin{array}{c}\text { Effet du GDF5-ßTCP } \\
\text { sur sinus lift vs } \\
\text { ßTCP-autogreffe }\end{array}$ & ßТСР & - & GDF-5 & - & $\begin{array}{l}\text { Surface de néoformation } \\
\text { osseuse sur biopsies test } \\
=\text { contrôle }\end{array}$ \\
\hline 48 & 1 cas & $\begin{array}{l}\text { Greffe en onlay sans } \\
\text { os autogène }\end{array}$ & TCP $\left(\right.$ Vitoss $\left.^{\circledR}\right)$ & - & rhBMP-2 & & $\begin{array}{l}\text { Succès: pose de } 6 \\
\text { implants après } 4 \text { mois }\end{array}$ \\
\hline
\end{tabular}

avec succès. Cependant, la grande variabilité des tableaux cliniques et le peu de cas publiés ne permettent pas de conclure à un apport particulier des cellules autologues dans cette indication.

Dans la régénération osseuse pré-implantaire ou parodontale, les cellules autologues ont été utilisées dans 17 cas sur 22. Dans 7 études [32,34-39], les ostéoblastes étaient issus d'un prélèvement de périoste, ce qui va dans le sens de procédures moins invasives (prélèvement buccal des cellules souches et amplification in vitro). Dans plusieurs cas, les cellules souches mésenchymateuses (d'origine médullaire, périostée ou pulpaire) induites vers un phénotype ostéoblastique ont permis d'obtenir une régénération osseuse significative (sinus lift [33], alvéoles d'extraction [40], augmentation transversale de la crête alvéolaire $[37,41]$, régénération périimplantaire [42] ou poches parodontales [39]). Les caractéristiques communes de ces défauts osseux sont d'une part leur volume limité et d'autre part l'absence de contraintes mécaniques appliquées pendant la cicatrisation. Plusieurs études rapportent des taux de succès implantaires proches de $100 \%$ [32, 35, 42-44] après des comblements sinusiens par des PIT.

Cependant, les résultats cliniques de l'utilisation de cellules souches mésenchymateuses autologues sont inconstants. Ainsi, plusieurs études utilisant des hydrogels cellularisés pour greffer des sinus ont révélé les limites de cette approche pour des comblements de volume important, en raison de la limite de diffusion de l'oxygène et des nutriments au sein 
des scaffolds [34, 36]. D'autre part, la résorption des volumes greffés par IT est parfois aussi importante, voire plus qu'avec l'os autogène $[45,46]$. Cela pourrait être lié à un temps de cicatrisation trop court ou bien à un volume initial très important avec peu d'os résiduel.

Les différences interindividuelles importantes concernant la quantité et la qualité des cellules souches mésenchymateuses peuvent aussi expliquer ces résultats inconstants. Enfin, toutes les sources de cellules mésenchymateuses ne sont pas équivalentes [60].

Les BMPs sont des protéines de la famille du TGFß qui jouent un rôle dans le recrutement, la prolifération et la différenciation des ostéoblastes [61]. L'utilisation des BMP2 et BMP-7 recombinantes a reçu une autorisation de mise sur le marché aux États-Unis et en Europe pour des indications bien précises en orthopédie (fractures ouvertes de la jambe, arthrodèses vertébrales), après plusieurs études prospectives chez l'Homme [62]. En chirurgie maxillo-faciale, quelques rapports de cas ont montré l'intérêt des BMPs dans la reconstruction mandibulaire après de larges résections osseuses $[10,17,63]$, en complément dans la chirurgie orthognathique [12] ou après des ostéomyélites [16-18,20]. Dans ces cas, des éponges de collagène, des allogreffes ou de la colle de fibrine étaient utilisés pour apporter sur le site les facteurs de croissance. Des taux de succès importants sont décrits, avec une grande quantité d'os néoformé de forte densité. La consolidation osseuse clinique apparaît rapidement (1 à 6 mois), même après radiothérapie ou lors d'une ostéomyélite [16-18]. Dans tous les cas de maturation in vivo, les BMPs (humaine, rhBMP-2, rhBMP-7) ont été utilisées en complément du scaffold pour favoriser le recrutement des cellules mésenchymateuses et leur différenciation en ostéoblastes. On remarque que les BMPs sont utilisées systématiquement dans les cas les plus difficiles de reconstruction osseuse (lésions étendues, terrain défavorable).

La principale complication rapportée de l'utilisation des BMPs est un œdème persistant plusieurs semaines après implantation dans quelques cas [16]. Certains essais ont décrit des taux d'échecs importants qui étaient liés à un manque de pureté des protéines utilisées (origine bovine) [11], à des difficultés opératoires ou à des scaffolds mal adaptés [16]. Les limites à l'utilisation des BMPs sont leur risque supposé de dissémination à distance du site d'implantation qui pourrait provoquer des proliférations cellulaires non contrôlées. Des stratégies de couplage des facteurs de croissance aux scaffolds ont été développées pour obtenir un effet strictement local [47]. Le coût important de ces traitements constitue également un obstacle pour une utilisation plus large.

La série de Chin et al. présente 50 cas de fentes labiales, palatines et faciales traitées par des éponges de collagène imprégnées de rhBMP-2 et par distraction osseuse. Un taux de succès de $98 \%$ (49 reconstructions osseuses sur 50) [30] est décrit, sans aucune autogreffe osseuse, ce qui a réduit de façon importante la morbidité. Cependant, des études randomisées à plus grande échelle sont nécessaires pour valider l'innocuité de ce protocole à long terme.
Dans les reconstructions pré-implantaires, l'utilisation de rhBMP-2 semble très efficace au niveau des sinus maxillaires $[31,48]$, mais également dans les greffes d'apposition $[48,49]$ et dans le comblement des ostéolyses périimplantaires [50]. La néoformation osseuse est rapide et se fait en grande quantité, de façon équivalente ou supérieure aux autogreffes [31]. Dans une étude prospective randomisée, la concentration efficace de rhBMP-2 en imprégnation d'éponges de collagène pour la régénération osseuse dans les sinus maxillaires a été évaluée à $1,5 \mathrm{mg} / \mathrm{mL}$ [51]. Dans une autre étude contrôlée randomisée pour le comblement de sinus, le greffage de rhGDF-5 sur un phosphate de calcium (BTCP) était équivalent au ßTCP non greffé associé à de l'os autogène [47]. Dans une étude prospective portant sur trois ans, concernant dix patients traités pour régénération osseuse pré-implantaire par des éponges de collagène imprégnées de rhBMP-2, aucune complication locale ou générale en rapport avec les BMPs n'a été observée [49].

Les BMPs ont été utilisées plus souvent dans les reconstructions mandibulaires que dans les régénérations osseuses pré-implantaires. Pour les reconstructions mandibulaires, les BMPs étaient utilisées en dernier recours, lorsque les méthodes conventionnelles (lambeaux) avaient échoué. Le bénéfice attendu de ces molécules était donc supérieur au risque éventuel lié à leur utilisation. Dans le cas des reconstructions pré-implantaires, les solutions alternatives plus invasives (greffes autologues) peuvent être préférables au risque éventuel encouru par l'utilisation de BMPs. De plus, le coût important de ces traitements constitue un frein à leur utilisation lorsque des solutions alternatives existent.

Deux méthodes de maturation différentes peuvent être utilisées en fonction de l'objectif recherché.

La maturation in vitro de courte durée (entre 4 et 7 jours) a été utilisée dans 1 cas pour la reconstruction mandibulaire, dans 1 cas pour la reconstruction de fentes faciales et dans 7 cas dans les régénérations osseuses pré-implantaires. Le but de cette étape était l'adhésion et la prolifération des cellules autologues sur le scaffold avant implantation. Dans certains cas, le temps de maturation in vitro était plus long (3 à 8 semaines) afin de permettre la sécrétion de la matrice extracellulaire et sa minéralisation. La maturation in vitro est donc essentiellement utilisée pour les PIT dans la régénération pré-implantaire parce qu'elle est peu invasive et que l'on recherche surtout un ensemencement des cellules du scaffold. Dans tous les cas, la maturation in vitro des PIT était faite en conditions statiques: il est probable que l'utilisation de bioréacteurs (conditions de culture dynamiques) pourrait améliorer la quantité et la répartition des cellules ensemencées dans les scaffolds, avec de meilleurs résultats cliniques [64].

À l'inverse, la maturation in vivo a uniquement été utilisée dans des reconstructions mandibulaires étendues car elle est beaucoup plus invasive et que l'objectif recherché est la mise en place d'une vascularisation du PIT avant implantation sur le site à reconstruire. Les cinq cas publiés ont tous abouti au développement d'une néovascularisation, objectivée par les examens complémentaires (scintigraphie ou angioscanner) après 2 à 8 mois de maturation, et confirmée in situ lors du 
transfert du lambeau. Le site de maturation in vivo était systématiquement au contact d'un muscle, dans une zone très vascularisée non concernée par les traitements antérieurs. Les conditions locales et générales du patient ont également une influence importante sur la cicatrisation. Après exérèse d'un kyste épidermoïde maxillaire récidivant, traité sans radiothérapie, Mesimäki et al. [4] ont obtenu une reconstruction mandibulaire satisfaisante mais Warnke et al. [6] rapportent un échec partiel dans la reconstruction mandibulaire après un cancer, traité par radiothérapie, chez un patient alcoolotabagique partiellement sevré.

La prévascularisation semble donc être une technique prometteuse dans les cas où les méthodes conventionnelles sont inefficaces en raison de conditions locales défaillantes et d'une insuffisance de la perfusion sanguine.

Dans deux études, la méthode des membranes induites a été utilisée pour une reconstruction mandibulaire. Le principal effet de la membrane est celui de mainteneur d'espace alors que les effets sur la prévention de la résorption du greffon et la sécrétion de facteurs ostéo-inducteurs par la membrane sont encore discutés $[22,65]$. L'état tissulaire local antérieur a également une influence sur le succès de cette technique car les suites opératoires ont été plus favorables avec les ostéomyélites (100\% de succès) [9] qu'avec les ostéoradionécroses (50\% de succès) [19].

\section{Conclusion}

Les applications de l'ingénierie tissulaire osseuse chez l'homme dans la sphère buccale et maxillo-faciale sont encore limitées et restent largement dans le champ de la recherche. L'IT présente deux indications principales dans ce domaine, avec des objectifs et des protocoles de traitement très différents.

Dans la reconstruction mandibulaire, les objectifs principaux sont la vascularisation du greffon mais également la stabilité primaire et la résistance mécanique à court terme des reconstructions. Les cas traités par IT étaient tous en échec thérapeutique avec les techniques conventionnelles. Des scaffolds mécaniquement résistants sont utilisés, avec des BMPs et éventuellement des méthodes de prévascularisation in vivo. Les cellules sont rarement utilisées dans cette indication car les résultats sont moins prédictibles qu'avec les BMPs. Les premières séries de cas de reconstruction mandibulaire ont montré des résultats encourageants mais des études complémentaires, comportant plus de cas, sont nécessaires pour confirmer ces premiers résultats.

À l'inverse, dans la régénération osseuse pré-implantaire, on recherche une moindre invasivité des procédures chirurgicales, notamment pour éliminer la morbidité des prélèvements d'autogreffes, tout en limitant le recours à des molécules aux effets secondaires potentiellement importants (BMPs). Les scaffolds n'ont pas besoin d'avoir une grande résistance mécanique mais ils doivent être malléables. Ils sont le plus souvent cellularisés in vitro avant implantation et les BMPs sont rarement utilisées. Dans cette seconde indication, l'ingénierie tissulaire a déjà montré des résultats similaires à ceux des méthodes classiques, ce qui ouvre la voie à des protocoles chirurgicaux moins invasifs.

\section{Conflits d'intérêt : aucun}

Remerciements. Les auteurs remercient l'Institut français pour la recherche odontologique (IFRO) pour son soutien financier.

\section{Références}

1. Langer R, Vacanti J. Tissue engineering. Science 1993;260:9206.

2. Lee KH. Tissue-engineered human living skin substitutes: development and clinical application. Yonsei Med J 2000;41:774-9.

3. Badylak SF, Nerem RM. Progress in tissue engineering and regenerative medicine. Proc Natl Acad Sci USA 2010;107:3285-6.

4. Mesimäki K, Lindroos B, Törnwall J, Mauno J, Lindqvist C, Kontio $R$, Miettinen $S$, Suururonen R. Novel maxillary reconstruction with ectopic bone formation by GMP adipose stem cells. Int J Oral Maxillofac Surg 2009;38:201-9.

5. Orringer JS, Shaw WW, Borud LJ, Freymiller EG, Wang SA, Markowitz BL. Total mandibular and lower lip reconstruction with a prefabricated osteocutaneous free flap. Plast Reconstr Surg 1999;104:793-7.

6. Warnke PH, Springer ING, Wiltfang $J$, Acil $Y$, Eufinger $H$, Wehmöller M, Russo PAJ, Bolte H, Sherry E, Behrens E, Terheyden $\mathrm{H}$. Growth and transplantation of a custom vascularised bone graft in a man. Lancet 2004;364:766-70.

7. Heliotis M, Lavery KM, Ripamonti U, Tsiridis E, Di Silvio L. Transformation of a prefabricated hydroxyapatite/osteogenic protein-1 implant into a vascularised pedicled bone flap in the human chest. Int J Oral Maxillofac Surg 2006;35:265-9.

8. Kokemueller H, Spalthoff S, Nolff M, Tavassol F, Essig H, Stuehmer C, Bornmann KH, Rücker M, Gellrich NC. Prefabrication of vascularized bioartificial bone grafts in vivo for segmental mandibular reconstruction: experimental pilot study in sheep and first clinical application. Int J Oral Maxillofac Surg 2010;39:379-87.

9. Goodger N, Wang J, Smagalski G, Hepworth B. Methylmethacrylate as a space maintainer in mandibular reconstruction. J Oral Maxillofac Surg 2005;63:1048-51.

10. Moghadam HG, Urist MR, Sandor GK, Clokie CM. Successful mandibular reconstruction using a BMP bioimplant. J Craniofac Surg 2001;12:119-27.

11. Ferretti C, Ripamonti U. Human segmental mandibular defects treated with naturally derived bone morphogenetic proteins. J Craniofac Surg 2002;13:434-44.

12. Warnke PH, Coren AJ. First experiences with recombinant human bone morphogenetic protein 7 (osteogenic protein 1) in a human case in maxillofacial surgery. Plast Reconstr Surg 2003;111:2471-2.

13. Warnke PH, Wiltfang J, Springer I, Acil Y, Bolte H, Kosmahl M, Russo P, Sherry E, Lützen U, Wolfart S, Terheyden H. Man as living bioreactor: fate of an exogenously prepared customized tissue-engineered mandible. Biomaterials 2006;27:3163-7. 
14. Pradel W, Eckelt U, Lauer G. Bone regeneration after enucleation of mandibular cysts: comparing autogenous grafts from tissueengineered bone and iliac bone. Oral Surg Oral Med Oral Pathol Oral Radiol Endod 2006;101:285-90.

15. Hibi H, Yamada Y, Kagami H, Ueda M. Distraction osteogenesis assisted by tissue engineering in an irradiated mandible: a case report. Int J Oral Maxillofac Implants 2006;21:141-7.

16. Carter TG, Brar PS, Tolas A, Beirne OR. Off-label use of recombinant human bone morphogenetic protein-2 (rhBMP-2) for reconstruction of mandibular bone defects in humans. J Oral Maxillofac Surg 2008; 66:1417-25.

17. Clokie CML, Sándor GKB. Reconstruction of 10 major mandibular defects using bioimplants containing BMP-7. J Can Dent Assoc 2008;74:67-72.

18. Herford AS, Boyne PJ. Reconstruction of mandibular continuity defects with bone morphogenetic protein-2 (rhBMP-2). J Oral Maxillofac Surg 2008;66:616-24.

19. Zwetyenga N, Catros S, Emparanza A, Deminiere C, Siberchicot F, Fricain JC. Mandibular reconstruction using induced membranes with autologous cancellous bone graft and HA-betaTCP: animal model study and preliminary results in patients. Int J Oral Maxillofac Surg 2009;38:1289-97.

20. Schuckert K, Jopp S, Teoh S. Mandibular defect reconstruction using three-dimensional polycaprolactone scaffold in combination with platelet-rich plasma and recombinant human bone morphogenetic protein-2: de novo synthesis of bone in a single case. Tissue Eng Part A 2009;15:493-9.

21. Lee J, Sung H, Jang J, Park Y, Min S, Kim E. Successful reconstruction of $15-\mathrm{cm}$ segmental defects by bone marrow stem cells and resected autogenous bone graft in central hemangioma. J Oral Maxillofac Surg 2010;68:188-94.

22. Catros S, Zwetyenga N, Bareille R, Brouillaud B, Renard M, Amédée J, Fricain JC. Subcutaneous induced membranes have no osteoinductive effect on macroporous ha-TCP in vivo. J Orthop Res 2008;27:155-61.

23. Masquelet AC, Fitoussi F, Begue T, Muller GP. Reconstruction of the long bones by the induced membrane and spongy autograft. Ann Chir Plast Esthet 2000;45:346-53.

24. Masquelet AC, Begue T. The concept of induced membrane for reconstruction of long bone defects. Orthop Clin North Am 2010;41:27-37.

25. Carstens MH, Chin M, Ng T, Tom WK. Reconstruction of \#7 facial cleft with distraction-assisted in situ osteogenesis (DISO): role of recombinant human bone morphogenetic protein-2 with Helistat-activated collagen implant. J Craniofac Surg 2005;16:1023-32.

26. Tom WK, Chin M, Ng T, Bouchoucha S, Carstens M. Distraction of rhBMP-2-generated mandible: how stable is the engineered bone in response to subsequent surgeries? J Oral Maxillofac Surg 2008;66:1499-505.

27. Hibi H, Yamada Y, Ueda M, Endo Y. Alveolar cleft osteoplasty using tissue-engineered osteogenic material. Int J Oral Maxillofac Surg 2006;35:551-5.

28. Pradel W, Tausche E, Gollogly J, Lauer G. Spontaneous tooth eruption after alveolar cleft osteoplasty using tissue-engineered bone: a case report. Oral Surg Oral Med Oral Pathol Oral Radiol Endod 2008;105:440-4.

29. Behnia H, Khojasteh A, Soleimani M, Tehranchi A, Khoshzaban A, Keshel SH, Atashi R. Secondary repair of alveolar clefts using human mesenchymal stem cells. Oral Surg Oral Med Oral Pathol Oral Radiol Endod 2009;108:e1-6.
30. Chin M, Ng T, Tom WK, Carstens M. Repair of alveolar clefts with recombinant human bone morphogenetic protein (rhBMP-2) in patients with clefts. J Craniofac Surg 2005;16:778-89.

31. Triplett RG, Nevins M, Marx RE, Spagnoli DB, Oates TW, Moy PK, Boyne PJ. Pivotal, randomized, parallel evaluation of recombinant human bone morphogenetic protein-2/absorbable collagen sponge and autogenous bone graft for maxillary sinus floor augmentation. J Oral Maxillofac Surg 2009;67:1947-60.

32. Beaumont C, Schmidt RJ, Tatakis DN, Zafiropoulos G. Use of engineered bone for sinus augmentation. J Periodontol 2008;79:541-8.

33. Mangano C, Piattelli A, Mangano A, Mangano F, Mangano A, Iezzi G, Borges F, d'Avila S, Shibli J. Combining scaffolds and osteogenic cells in regenerative bone surgery: a preliminary histological report in human maxillary sinus augmentation. Clin Implant Dent Relat Res 2009;11:e92-102.

34. Voss P, Sauerbier S, Wiedmann-Al-Ahmad M, Zizelmann C, Stricker A, Schmelzeisen R, Gutwald R. Bone regeneration in sinus lifts: comparing tissue-engineered bone and iliac bone. $\mathrm{Br}$ J Oral Maxillofac Surg 2010;48:121-6.

35. Schmelzeisen R, Schimming R, Sittinger M. Making bone: implant insertion into tissue-engineered bone for maxillary sinus floor augmentation-a preliminary report. J Craniomaxillofac Surg 2003:31:34-9.

36. Schimming R, Schmelzeisen R. Tissue-engineered bone for maxillary sinus augmentation. J Oral Maxillofac Surg 2004;62:724-9.

37. Strietzel FP. Tissue-engineered bone for lateral alveolar ridge augmentation: a case report. Int J Oral Maxillofac Implants 2006;21:131-5

38. Springer IN, Nocini PF, Schlegel KA, De Santis D, Park J, Warnke $\mathrm{PH}$, Terheyden H, Zimmermann R, Chiarini L, Gardner K, Ferrari F, Wiltfang J. Two techniques for the preparation of cell-scaffold constructs suitable for sinus augmentation: steps into clinical application. Tissue Eng 2006;12:2649-56.

39. Yamamiya K, Okuda K, Kawase T, Hata K, Wolff LF, Yoshie H. Tissue-engineered cultured periosteum used with platelet-rich plasma and hydroxyapatite in treating human osseous defects. J Periodontol 2008;79:811-8.

40. d'Aquino R, De Rosa A, Lanza V, Tirino V, Laino L, Graziano A, Desiderio V, Laino G, Papaccio G. Human mandible bone defect repair by the grafting of dental pulp stem/progenitor cells and collagen sponge biocomplexes. Eur Cell Mater 2009;18:75-83.

41. Kulakov AA, Goldshtein DV, Grigoryan AS, Rzhaninova AA, Alekseeva IS, Arutyunyan IV, Volkov AV. Clinical study of the efficiency of combined cell transplant on the basis of multipotent mesenchymal stromal adipose tissue cells in patients with pronounced deficit of the maxillary and mandibulary bone tissue. Bull Exp Biol Med 2008;146:522-5.

42. Yamada Y, Nakamura S, Ito K, Kohgo T, Hibi H, Nagasaka T, Ueda M. Injectable tissue-engineered bone using autogenous bone marrow-derived stromal cells for maxillary sinus augmentation: clinical application report from a 2-6-year follow-up. Tissue Eng Part A 2008;14:1699-707.

43. Shayesteh YS, Khojasteh A, Soleimani M, Alikhasi M, Khoshzaban A, Ahmadbeigi N. Sinus augmentation using human mesenchymal stem cells loaded into a beta-tricalcium phosphate/hydroxyapatite scaffold. Oral Surg Oral Med Oral Pathol Oral Radiol Endod 2008;106:203-9.

44. Ueda M, Yamada $Y$, Kagami H, Hibi H. Injectable bone applied for ridge augmentation and dental implant placement: human progress study. Implant Dent 2008;17:82-90. 
45. Zizelmann C, Schoen R, Metzger MC, Schmelzeisen R, Schramm A, Dott B, Bormann K, Gellrich N. Bone formation after sinus augmentation with engineered bone. Clin Oral Implants Res 2007; 18:69-73.

46. Fuerst G, Strbac GD, Vasak C, Tangl S, Leber J, Gahleitner A, Gruber R, Watzek G. Are culture-expanded autogenous bone cells a clinically reliable option for sinus grafting? Clin Oral Implants Res 2009;20:135-9.

47. Koch FP, Becker J, Terheyden H, Capsius B, Wagner W. A prospective, randomized pilot study on the safety and efficacy of recombinant human growth and differentiation factor- 5 coated onto beta-tricalcium phosphate for sinus lift augmentation. Clin Oral Implants Res 2010 Jun 8 [Epub ahead of print].

48. Schuckert K, Jopp S, Osadnik M. Modern bone regeneration instead of bone transplantation: a combination of recombinant human bone morphogenetic protein-2 and platelet-rich plasma for the vertical augmentation of the maxillary bone-A single case report. Tissue Eng Part C Methods 2010 Apr 26 [Epub ahead of print].

49. Cochran DL, Jones AA, Lilly LC, Fiorellini JP, Howell $H$. Evaluation of recombinant human bone morphogenetic protein2 in oral applications including the use of endosseous implants: 3-year results of a pilot study in humans. J Periodontol 2000;71:1241-57.

50. Jung RE, Glauser R, Schärer P, Hämmerle CHF, Sailer HF, Weber $F E$. Effect of rhBMP-2 on guided bone regeneration in humans. Clin Oral Implants Res 2003;14:556-68.

51. Boyne PJ, Lilly LC, Marx RE, Moy PK, Nevins M, Spagnoli DB, Triplett RG. De novo bone induction by recombinant human bone morphogenetic protein-2 (rhBMP-2) in maxillary sinus floor augmentation. J Oral Maxillofac Surg 2005;63:1693-707.

52. Yamada Y, Ueda M, Hibi H, Nagasaka T. Translational research for injectable tissue-engineered bone regeneration using mesenchymal stem cells and platelet-rich plasma: from basic research to clinical case study. Cell Transplant 2004;13:343-55.

53. Meijer GJ, de Bruijn JD, Koole R, van Blitterswijk CA. Cell based bone tissue engineering in jaw defects. Biomaterials 2008;29:3053-61.

54. Haute Autorité de Santé. Guide d'analyse de la littérature et gradation des recommandations 2000.

55. Baqain ZH, Anabtawi M, Karaky AA, Malkawi Z. Morbidity from anterior iliac crest bone harvesting for secondary alveolar bone grafting: an outcome assessment study. J Oral Maxillofac Surg 2009;67:570-5.
56. Schaaf H, Lendeckel S, Howaldt H, Streckbein P. Donor site morbidity after bone harvesting from the anterior iliac crest. Oral Surg Oral Med Oral Pathol Oral Radiol Endod 2010;109:52-8.

57. Boyapati L, Wang $H$. The role of platelet-rich plasma in sinus augmentation: a critical review. Implant Dent 2006;15:160-70.

58. Marx RE. Platelet-rich plasma: evidence to support its use. J Oral Maxillofac Surg 2004;62:489-96.

59. Gimbel M, Ashley RK, Sisodia M, Gabbay JS, Wasson KL, Heller J, Wilson L, Kawamoto H, Bradley J. Repair of alveolar cleft defects: reduced morbidity with bone marrow stem cells in a resorbable matrix. J Craniofac Surg 2007;18:895-901.

60. Mosna F, Sensebé L, Krampera M. Human bone-marrow and adipose tissue mesenchymal stem cells: a user's guide. Stem Cells Dev 2010; sous presse.

61. Ebara S, Nakayama K. Mechanism for the action of bone morphogenetic proteins and regulation of their activity. Spine 2002;27:S10-5.

62. Govender S, Csimma C, Genant HK, Valentin-Opran A, Amit Y, Arbel R, Aro H, Atar D, Bishay M, Börner MG, Chiron P, Choong P, Cinats J, Courtenay B, Feibel R, Geulette B, Gravel C, Haas $N$, Raschke M, Hammacher E, van der Velde D, Hardy P, Holt M, Josten C, Ketterl RL, Lindeque B, Lob G, Mathevon H, McCoy G, Marsh D, Miller R, Munting E, Oevre S, Nordsletten L, Patel A, Pohl A, Rennie W, Reynders P, Rommens PM, Rondia J, Rossouw WC, Daneel PJ, Ruff S, Rüter A, Santavirta S, Schildhauer TA, Gekle C, Schnettler R, Segal D, Seiler H, Snowdowne RB, Stapert J, Taglang G, Verdonk R, Vogels L, Weckbach A, Wentzensen A, Wisniewski T. Recombinant human bone morphogenetic protein2 for treatment of open tibial fractures: a prospective, controlled, randomized study of four hundred and fifty patients. J Bone Joint Surg Am 2002;84:2123-34.

63. Chao M, Donovan T, Sotelo C, Carstens MH. In situ osteogenesis of hemimandible with rhBMP-2 in a 9-year-old boy: osteoinduction via stem cell concentration. J Craniofac Surg 2006;17:40512.

64. Martin I, Wendt D, Heberer M. The role of bioreactors in tissue engineering. Trends Biotechnol 2004;22:80-6.

65. Pelissier P, Masquelet AC, Bareille R, Pelissier SM, Amedee J. Induced membranes secrete growth factors including vascular and osteoinductive factors and could stimulate bone regeneration. J Orthop Res 2004;22:73-9. 